\title{
Physical fitness in Spanish naval cadets. A four-year study
}

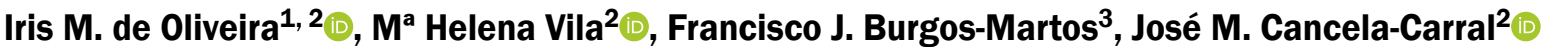

\author{
${ }^{1}$ Department of Functional Biology and Health Sciences, University of Vigo, Spain \\ ${ }^{2}$ HealthyFit Research Group, Galicia Sur Health Research Institute (IIS Galicia Sur), Sergas-UVIGO, Spain \\ ${ }^{3}$ Department of Physical Education, Military Naval Academy in Marín, Spain
}

\begin{abstract}
Background: Military physical readiness largely depends on soldiers maintaining their general health and physical fitness at a level which enables them to perform their requisite occupational duties in garrison and deployment environments. Understanding the physical fitness of naval cadets throughout its formative stages will help us define a training programme tailored to their needs. The objective of this study was to analyse the physical fitness of cadets enrolled in military training in the academic year 2018-2019 in the Military Naval Academy in Marín, Spain.

Materials and methods: A longitudinal correlational-descriptive design was undertaken. 167 of the 292 students who participated in the global analysis participated in the pre and post (first and second semester) physical fitness tests $(153$ males and 14 females, mean age $21.9 \pm 3.5)$ and were stratified into age groups. Anthropometric measurements and data from the Spanish army physical fitness assessment system ( 2 min push-ups, 1000 and $50 \mathrm{~m}$ races, vertical jump and $50 \mathrm{~m}$ swimming) were used. A descriptive, inferential, and correlational analysis was carried out and the level of significance set for the study was $p \leq 0.05$.

Results: Inferential analysis between age group markers and over the total sample shows statistical differences for the total sample in the $1000 \mathrm{~m}$ and $50 \mathrm{~m}$ race $(p<0.001)$ and in vertical jump test $(p<0.010)$. There was significant statistical correlation between all five tests undertaken.

Conclusions: The level of physical fitness of cadets at the Military Naval Academy in Marin, Spain appears to be acceptable when compared to other countries' military naval corps. Coherence between training and evaluation is extremely important, and a re-evaluation of stamina and strength training is probably advisable.

(Int Marit Health 2021; 72, 1: 10-17)
\end{abstract}

Key words: physical fitness, military personnel, physical conditioning, athletic performance

\section{INTRODUCTION}

Military physical readiness largely depends on soldiers maintaining their general health and physical fitness at a level which enables them to perform their requisite occupational duties in garrison and deployment environments [1-3]. A physically capable force is required to perform essential military tasks. The importance of physical fitness applies to the general population, but for military personnel, achieving a high level of physical fitness may be essential for them to be successful at their jobs [4-6]. Additionally, combat deployments may last for extended periods, and soldiers are expected to maintain their levels of fitness during these times. Maintaining fitness during deployment may be achieved by the physical requirements and demands of the soldier's role and duties, or through the implementation of structured physical training. Military units typically train

Dr. José Ma Cancela, Faculty of Education and Sports Science, University of Vigo (Spain), Campus a Xunqueira, s/n, 36005 Pontevedra, Spain, tel: 986801700 ,

fax: 986801701, e-mail: chemacc@uvigo.es

The project has been developed in the Military Naval Academy in Marín, Spain.

This article is available in open access under Creative Common Attribution-Non-Commercial-No Derivatives 4.0 International (CC BY-NC-ND 4.0) license, allowing to download articles and share them with others as long as they credit the authors and the publisher, but without permission to change them in any way or use them commercially. 
as a group when carrying out physical training. Training is used not only for the physical conditioning necessary for the demands of the job, but also for stimulating esprit de corps, teamwork, camaraderie, and mental resilience [7].

The training of new recruits, including their development of the physical capacities necessary for them to be effective operational soldiers, places important emphasis on military training systems and curricula. Furthermore, these training systems and curricula must also be sufficiently robust to ensure the satisfactory progression of recruits' or cadets' fitness within a set period of time [8,9]. Each branch of the armed forces determines the necessary physical fitness testing needed to maintain minimal levels of fitness and strength for its members' respective duties [6, 10-12]. Physical fitness assessments are an essential component for the measurement of the physical capacities of all Service Members [1]. For example, in the United States of America (USA), soldiers must meet certain fitness criteria to remain in the military. The Army Physical Fitness Test (APFT) is taken twice a year by the active duty population, and is the tool used to assess physical fitness. It has three components: extended-leg push-ups, bent-knee sit-ups and a timed 2-mile run [13].

The importance of adequate levels of physical fitness is emphasized in military personnel due to the high physical demands which exist during military training and in warfare [10, 11]. In the USA, soldiers who fail the APFT usually participate in remedial physical training programmes, while repeated failures can lead to their discharge from military service [13].

Physical exercise/sports assignments should be oriented towards individual personal conditioning. Although leaders acknowledge that physical fitness tests are part of military service, those tests' marks are not a proven predictor of adequate military physical fitness, and the specificity of performance-related fitness requirements should vary depending on the individual's mission [10]. It is important to highlight that in Spain, different Military Corps use different tests to assess the physical fitness of military personnel, and that currently, no battery of tests has been scientifically validated. Furthermore, in Spain, each of the armed services conducts some variation of an annual or biannual physical test. These periodic physical tests carried out are in accordance with the Spanish Ministerial Order 54/2014, November $11^{\text {th }}$.

Based on the comments above, the objective of this study was to report the level of physical fitness of Spanish cadets enrolled in the Bachelor's degree and military programme for 4 years.

\section{MATERIALS AND METHODS}

This study has a quantitative approach and uses a longitudinal correlational-descriptive design, and it is part of a longitudinal study in collaboration with Military Naval
Academy in Marín (MNAM), Spain from 2018 to 2022 under the "Admiral Álvarez-Ossorio" endowed chair.

The sample included students enrolled in the Bachelor's degree and military training programme of 5 year's duration, at the MNAM attached to the University of Vigo during the academic year 2018/2019. One hundred and sixty-seven of the 292 students who participated in the global analysis participated in the pre and post physical fitness tests and have consequently been included in the study sample (153 males and 14 women, mean age $21.9 \pm 3.5$ ).

The anthropometric assessment was carried out by specialised technicians and took place in the multi-use room the week before the physical tests. The anthropometric assessment followed the International Working Group of Kinanthropometry (ISAK) protocol [14]. The instrument included students' weight, height, and body mass index (BMI). Height was measured using a telescopic measuring rod SECA (SECA, Germany) with a measuring accuracy of $0.1 \mathrm{~cm}$. Weight was measured using a bioimpedance device Tanita (TBF300) with a measuring accuracy of $100 \mathrm{~g}$. For the body composition study, the percentage of fat was calculated based on the BMI, using the following formula: weight/height ${ }^{2}\left(\mathrm{~kg} / \mathrm{m}^{2}\right)$.

The Spanish army physical fitness assessment system provides information about the physical activity training programme and the recruit's current level of fitness. This assessment allows an individual physical profile to be defined, including the three groups correspondent to the following aspects of physical condition: strength, stamina, and speed. This instrument includes the General Physical Condition Test and the Specific Physical Condition Test. Physical fitness assessment (Supplementary Material - see journal website) was carried out using different tests included in the General Physical Condition Test [15].

Assessments were carried out in three different locations: on an athletics track (50 $\mathrm{m}$ race and $1000 \mathrm{~m}$ race), in a multi-use room (vertical jump and 2 min push ups) and in an indoor swimming pool (50 m swimming). The first assessment was carried out over a week, in the mornings from 8:00 to 9:30, in January. The second assessment was also carried out for a week, in the mornings, from 12:30 to 14:0, in May. The first day the $1000 \mathrm{~m}$ race was run, the second day the $50 \mathrm{~m}$ swimming, the third day $2 \mathrm{~min}$ push ups and vertical jump, the fourth day the $50 \mathrm{~m}$ race and on the fifth day those participants who could not take part on the previous days were assessed. Anthropometric assessment was carried out in the multi-use room the week before the physical tests. Before the physical fitness tests, participants warmed up for $15 \mathrm{~min}$ in accordance with the tests to be done. The physical fitness tests were carried out by military personnel of the Physical Activity Department, professionals who are used to recording the data of such 
Table 1. Descriptive analysis by course

\begin{tabular}{llllll}
\hline & All course $(\mathbf{n}=\mathbf{1 6 7})$ & $\mathbf{1}^{\text {st }}$ course $(\mathbf{n}=\mathbf{6 9})$ & $\mathbf{2}^{\text {nd }}$ course $(\mathbf{n}=\mathbf{4 4})$ & $\mathbf{3}^{\text {rd }}$ course $(\mathbf{n}=\mathbf{2 7})$ & $\mathbf{4}^{\text {th }}$ course $(\mathbf{n}=\mathbf{2 7})$ \\
\hline Gender $(\%$ male) & $91.7 \%$ & $91.8 \%$ & $86.8 \%$ & $97.2 \%$ & $87.9 \%$ \\
Age [years] & $21.9 \pm 0.9$ & $18.6 \pm 0.5$ & $20.5 \pm 0.5$ & $22.3 \pm 0.5$ & $27.7 \pm 3.2$ \\
Height [cm] & $176.1 \pm 7.4$ & $176.2 \pm 7.2$ & $175.2 \pm 7.8$ & $178.0 \pm 7.0$ & $174.7 \pm 7.9$ \\
Weight [kg] & $73.4 \pm 7.9$ & $712 \pm 8.4$ & $72.1 \pm 9.1$ & $77.1 \pm 9.3$ & $73.5 \pm 9.0$ \\
BMl [kg/m²] & $23.6 \pm 2.1$ & $22.9 \pm 2.0$ & $23.4 \pm 2.2$ & $24.3 \pm 2.2$ & $24.0 \pm 2.0$ \\
1000 m race [min] & $03: 22.3 \pm 00: 15.0$ & $03: 23.3 \pm 00: 14.4$ & $03: 22.0 \pm 00: 16.5$ & $03: 17.4 \pm 00: 13.0$ & $03: 30.3 \pm 00: 15.7$ \\
50 m race [s] & $7.0 \pm 0.5$ & $7.0 \pm 0.5$ & $6.9 \pm 0.5$ & $6.8 \pm 0.4$ & $7.1 \pm 0.51$ \\
2 min push ups & $42.7 \pm 11.8$ & $42.8 \pm 10.8$ & $42.5 \pm 12.0$ & $42.6 \pm 11.1$ & $42.7 \pm 10.7$ \\
(repetitions) & & & & & $53.4 \pm 5.7$ \\
Vertical jump test & $55.2 \pm 5.8$ & $53.5 \pm 6.9$ & $55.7 \pm 7.4$ & & \\
[cm] & & $37.7 \pm 4.9$ & $38.3 \pm 7.8$ & $22.3 \pm 0.5$ & $39.0 \pm 5.7$ \\
50 m swimming [s] & $37.8 \pm 7.6$ & & &
\end{tabular}

physical fitness tests. Anthropometric assessment was carried out by specialized technicians.

A descriptive statistical analysis based on the data has been carried out using central tendency measures and deviations, as this analysis is stratified by age. To verify the data for normality, the Shapiro-Wilks test was used ( $p>0.05$ ). To determine if the training programme had a significant effect on the anthropometric and physical fitness variables, a Student $t$ test for paired data was used. With a view to identifying the associations between the physical fitness tests which were used for both first and second semesters, a correlational analysis was carried out. Statistical analysis was performed using IBM-SPSS v25 for Mac. The level of significance was $p<0.05$.

\section{ETHICS APPROVAL AND CONSENT TO PARTICIPATE}

This study is in accordance with the Helsinki Declaration with respect to data collection and processing (World Medical Association, 2013) and with the Spanish Ley Orgánica de Protección de Datos de Carácter Personal. This protocol was submitted for consideration, commenting, guidance and approval to the research ethics committee of Faculty of Sports and Education Sciences (University of Vigo) and accepted before the study began with the code number 03-719.

\section{RESULTS}

Table 1 shows descriptive analysis and sample characteristics by age groups. The total sample was composed of 167 students between 18 and 37 years old.

Inferential analysis between the results obtained in the first and second semesters by age group shows statistical differences for the total sample in the $1000 \mathrm{~m}$ and $50 \mathrm{~m}$ race $(p<0.001)$ and in the vertical jump test $(p<0.010)$
(Table 2). Depending on the age group analysed, statistical differences were observed, such as in the 2 min push ups ( $p<0.001)$ for the 18-19 age group; whereas in the 20-21 age group no statistical differences were observed for the vertical jump test, and in the $>24$ age group no differences were observed for the $1000 \mathrm{~m}$ race.

Table 3 summarises the physical activity undertaken in the first and second semester of the academic year 2018-2019. It should be noted that the physical activity planning used at MNAM includes characteristics intended to train and develop those physical fitness aspects (upper limb strength, general stamina, velocity, lower limb strength and swimming performance) associated with the Spanish army physical fitness assessment system.

A correlational analysis between the results obtained in the physical fitness tests of the first and second semester indicates a statistical correlation between all the five tests carried out. There was a significant statistical positive correlation between the $1000 \mathrm{~m}$ race, $50 \mathrm{~m}$ race and $50 \mathrm{~m}$ swimming, and between the 2 min push ups and vertical jump tests, while a significant statistical negative correlation was observed between the $1000 \mathrm{~m}$ race, $50 \mathrm{~m}$ race and 50 m swimming, and the last two tests: 2 min push ups and vertical jump test $(p<0.001)$. Finally, Figure 1 shows the $\mathrm{VO}_{2}$ max evolution by age group, calculated based on the results of the $1000 \mathrm{~m}$ race, and comparing measurements carried out in the first and second semesters.

\section{DISCUSSION}

The objective of this study was to analyse the physical fitness of the cadets enrolled in the five years duration of the Bachelor's degree and military training. Our sample includes a real representation of gender percentages be- 
Table 2. Inferential analysis between first and second semester physical fitness tests

\begin{tabular}{|c|c|c|c|c|c|c|c|c|c|}
\hline & & \multicolumn{5}{|c|}{ Mean of the differences } & \multirow[t]{3}{*}{$\mathbf{t}$} & \multirow[t]{3}{*}{ gl } & \multirow[t]{3}{*}{$\mathbf{p}$} \\
\hline & & \multirow[t]{2}{*}{ Mean } & \multirow[t]{2}{*}{ SD } & \multirow[t]{2}{*}{ Error } & \multicolumn{2}{|l|}{$\mathrm{Cl}(95 \%)$} & & & \\
\hline & & & & & Lower & Higher & & & \\
\hline \multirow{5}{*}{$\begin{array}{l}\text { All } \\
\text { course }\end{array}$} & $1000 \mathrm{~m}$ race $[\mathrm{min}]$ & 00:03.8 & $00: 08.2$ & $00: 00.6$ & $00: 02.6$ & 00:05.1 & 6.1 & 167 & 0.001 \\
\hline & $50 \mathrm{~m}$ race $[\mathrm{s}]$ & -0.2 & 0.3 & 0.1 & -0.2 & -0.1 & -7.9 & 167 & 0.001 \\
\hline & 2 min push ups [reps] & -0.7 & 6.9 & 0.5 & -1.7 & 0.4 & -1.2 & 163 & 0.229 \\
\hline & Vertical jump test [cm] & -0.9 & 4.0 & 0.3 & -1.5 & -0.3 & -2.9 & 163 & 0.004 \\
\hline & 50 m swimming [s] & 0.1 & 2.5 & 0.2 & -0.3 & 0.4 & 0.2 & 163 & 0.827 \\
\hline \multirow{5}{*}{$\begin{array}{l}1^{\text {st }} \\
\text { course }\end{array}$} & $1000 \mathrm{~m}$ race [min] & 00:07.6 & 00:06.2 & $00: 00.7$ & 00:06.1 & 00:09.0 & 10.3 & 69 & 0.001 \\
\hline & $50 \mathrm{~m}$ race $[\mathrm{s}]$ & -0.1 & 0.3 & 0.1 & -0.2 & -0.1 & -4.2 & 69 & 0.001 \\
\hline & 2 min push ups [reps] & -1.9 & 6.7 & 0.8 & -3.5 & -0.3 & -2.4 & 68 & 0.022 \\
\hline & Vertical jump test [cm] & -2.2 & 3.8 & 0.5 & -3.1 & -1.2 & -4.7 & 67 & 0.001 \\
\hline & 50 m swimming [s] & 0.2 & 2.2 & 0.3 & -0.4 & 0.7 & 0.6 & 69 & 0.561 \\
\hline \multirow{5}{*}{$\begin{array}{l}2^{\text {nd }} \\
\text { course }\end{array}$} & $1000 \mathrm{~m}$ race [min] & 00:03.8 & $00: 07.4$ & 00:01.1 & 00:01.5 & 00:06.0 & 3.4 & 43 & 0.001 \\
\hline & $50 \mathrm{~m}$ race $[\mathrm{s}]$ & -0.2 & 0.3 & 0.1 & -0.3 & -0.1 & -4.3 & 43 & 0.001 \\
\hline & 2 min push ups [reps] & -0.6 & 7.1 & 1.1 & -2.9 & 1.6 & -0.6 & 40 & 0.572 \\
\hline & Vertical jump test [cm] & -0.9 & 4.2 & 0.6 & -2.2 & 0.4 & -1.4 & 41 & 0.181 \\
\hline & 50 m swimming [s] & 0.4 & 3.3 & 0.5 & -0.7 & 1.4 & 0.7 & 40 & 0.479 \\
\hline \multirow{5}{*}{$\begin{array}{l}3^{\text {rd }} \\
\text { course }\end{array}$} & $1000 \mathrm{~m}$ race [min] & 00:04.3 & 00:07.3 & 00:01.4 & 00:07.1 & 00:01.4 & -3.1 & 26 & 0.005 \\
\hline & $50 \mathrm{~m}$ race $[\mathrm{s}]$ & -0.2 & 0.2 & 0.1 & -0.3 & -0.1 & -4.1 & 26 & 0.001 \\
\hline & 2 min push ups [reps] & -0.2 & 6.3 & 1.2 & -2.7 & 2.3 & -0.2 & 26 & 0.879 \\
\hline & Vertical jump test [cm] & 1.6 & 3.5 & 0.7 & 0.2 & 3.0 & 2.3 & 26 & 0.031 \\
\hline & $50 \mathrm{~m}$ swimming [s] & -0.3 & 1.7 & 0.3 & -0.9 & 0.4 & -0.8 & 26 & 0.429 \\
\hline \multirow{5}{*}{$\begin{array}{l}4^{\text {th }} \\
\text { course }\end{array}$} & $1000 \mathrm{~m}$ race $[\mathrm{min}]$ & $00: 02.6$ & 00:09.1 & $00: 01.7$ & 00:00.9 & 00:06.2 & 1.5 & 26 & 0.142 \\
\hline & $50 \mathrm{~m}$ race $[\mathrm{s}]$ & -0.2 & 0.2 & 0.1 & -0.3 & -0.1 & -3.8 & 26 & 0.001 \\
\hline & 2 min push ups [reps] & 2.1 & 6.9 & 1.3 & -0.7 & 4.8 & 1.6 & 26 & 0.132 \\
\hline & Vertical jump test [cm] & -0.2 & 3.5 & 0.7 & -1.6 & 1.2 & -0.3 & 26 & 0.747 \\
\hline & 50 m swimming [s] & -0.5 & 2.4 & 0.5 & -1.5 & 0.5 & -0.9 & 25 & 0.343 \\
\hline
\end{tabular}

$\mathrm{Cl}$ - confidence interval; SD - standard deviation

tween groups in MNAM. The total sample participating in the study was 167 as mentioned in the "Results" section, but only $8.3 \%$ were women. Men and women involved in military service carry out jobs that require a high level of physical fitness irrespective of their age, rank, or role [4]. Additionally, the physical conditioning of the sample improves with age and the best results were observed in the subgroup of 22-23 years.

Scientific evidence maintains that the response of physiological systems to exercise is specific to the mode and intensity of exercise, and that the training programme undertaken must stress the physiological systems that are critical for optimal performance in the given task so as to achieve specific training adaptations. There are a variety of fitness assessment tests available for measuring performance or changes in fitness [16].
The training protocol carried out by MNAM cadets may seem timely adjusted for daily training time (50 min per day), which might lead to the idea that it might compromise obtaining the minimum marks required in the different fitness components of the test battery (minimum marks required with increment as the academic course of study progresses). Another aspect to keep in mind is that the trainings are mostly carried out in a group form, and not necessarily organized based on the homogeneity of physical condition of the cadets as well as in other armies [7].

Cuddy et al. [4] in their study conclude that candidates for entry into some Special Operations Forces in the USA who complete 30 min per day or more of physical activity have a higher likelihood of passing the physical fitness tests, or at least the run portion. Because the run portion is more 
Table 3. Resume of the physical activity programme developed during the first and second semester of the academic course 2018-2019

\begin{tabular}{|c|c|c|c|c|}
\hline Monday & Tuesday & Wednesday & Thursday & Friday \\
\hline \multicolumn{5}{|l|}{$1^{\text {st }}$ Semester } \\
\hline $\begin{array}{l}\text { Fartlek run } \\
\text { Warming: } \\
\text { 7' continuous run + running } \\
\text { technique, high skipping, } \\
\text { heels back, tips of toes above, } \\
\text { extended knees } \\
\text { Main part: } \\
\text { Fartlek run: } 5 \text { series } \\
\text { Density 1:1 } \\
\text { Passive recovering R0 } \\
\text { R5 15" } \\
\text { R4 25" } \\
\text { R3 50" } \\
\text { R2 } 60 " \\
\text { Back to calm: } \\
\text { Stretching }\end{array}$ & $\begin{array}{l}\text { Continuous run } \\
\text { Warming: } \\
\text { 5'R1 } \\
\text { Main part: } \\
\text { 25'R2 } \\
\text { Back to calm: } \\
\text { Stretching }\end{array}$ & $\begin{array}{l}\text { Swimming } \\
\text { Warming: } \\
\text {-200 m light aerobic rhythm (100 } \\
\text { FC+66 Ba+33 Br) (15”) } \\
\text { Main part: } \\
\text { Progressive: } \\
\text {-200 m 66×3 Prog. 1-3 FC (30”) } \\
\text {-100 m 33×3 Prog. 1-3 Ba (30") } \\
\text {-100 m 33×3 Prog. 1-3 Br (30”) } \\
\text { Regressive: } \\
\text {-200 m } 66 \times 3 \text { Regr. 1-3 FC (30") } \\
\text {-100 m 33×3 Regr. 1-3 Ba (30”) } \\
\text {-100 m 33×3 Regr. 1-3 Br (30”) } \\
\text { Back to calm: } \\
\text {-100-200 m smooth freestyle } \\
\text { Total: } 1100-1200 \text { m }\end{array}$ & $\begin{array}{l}\text { Specific slopes } \\
\text { Warming: } \\
\text { 15' continuous } \\
\text { run, including se- } \\
\text { ries section once } \\
\text { Main part: } \\
2 \times(3 \times 250 \text { m in } \\
\text { slope R3 + 2' Re- } \\
\text { covering) 4' Rec. } \\
\text { Back to calm: } \\
\text { Stretching }\end{array}$ & $\begin{array}{l}\text { Power circuit } \\
\text { Warming: } \\
\text { 5' continuous run + rhythmic } \\
\text { lap } 250 \text { m } \\
\text { Main part: } \\
\text { Interval training 10' } 3 \times \\
\text { (30':15') 1'rec passive } \\
\text { Isometric trunk flexion } \\
\text { Oblique touching floor } \\
\text { Abdominal scissors } \\
\text { Circuit } 2 \times \text { (8' maximum } \\
\text { repetitions) 5' rec. Passive } \\
30 \text { Flexions } \\
20 \text { Squats } \\
10 \text { Triceps funds } \\
5 \text { Shoulder flexions } \\
250 \text { m continuous running } \\
\text { Back to calm: } \\
\text { Stretching }\end{array}$ \\
\hline \multicolumn{5}{|l|}{$2^{\text {nd }}$ Semester } \\
\hline 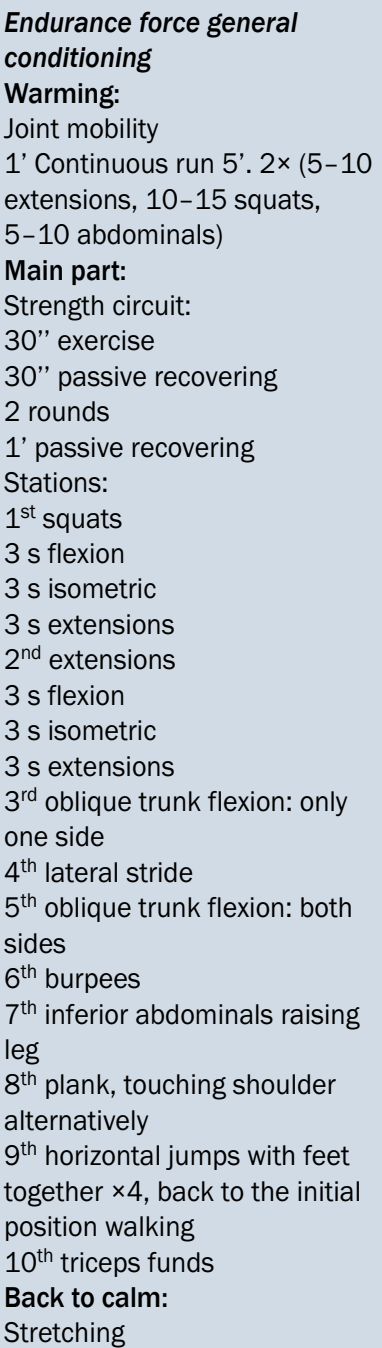 & $\begin{array}{l}\text { Medium interval } \\
\text { training } \\
\text { Warming: } \\
10 \text { ' continuous } \\
\text { run } \\
2 \text { progressive } \\
30 \text { m } \\
\text { Recovering } \\
\text { walking back } \\
\text { Main part: } \\
2 \times \text { (3× } 200 \text { m } \\
\text { R4 2'Rec. R0) } \\
4 \text { 'Rec R0 } \\
\text { It is sought to } \\
\text { reach a constant } \\
\text { brand for the } \\
\text { repetitions. Do } \\
\text { not do the first at } \\
\text { maximum effort } \\
\text { and progressi- } \\
\text { vely improving } \\
\text { the following } \\
\text { repetitions } \\
\text { Back to calm: } \\
\text { Stretching }\end{array}$ & 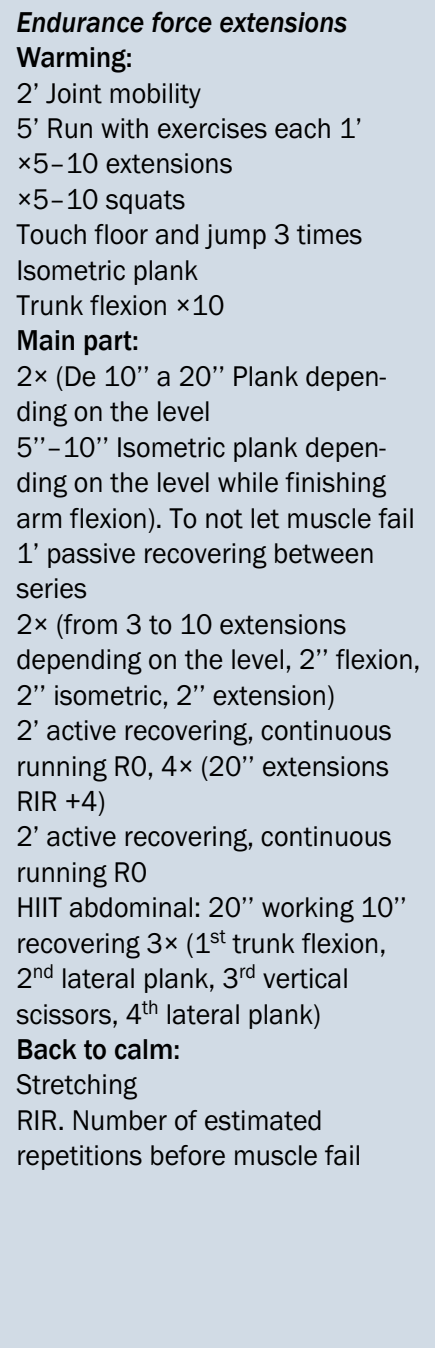 & $\begin{array}{l}\text { Aerobic slopes } \\
\text { Warming: } \\
\text { 10' continuous } \\
\text { running } \\
2 \text { progressives } \\
30 \text { m } \\
\text { Recovering wal- } \\
\text { king back } \\
\text { Main part: } \\
2 \times \text { ( } 2 \times 200 \text { m } \\
\text { in slope R4 3' } \\
\text { recovering R0) } \\
\text { 4' Recovering R0 } \\
\text { Back to calm: } \\
\text { Stretching }\end{array}$ & $\begin{array}{l}\text { Running technique } \\
\text { Warming: } \\
5^{\prime} \text { continuous running } \\
\text { Main part: } \\
\text { Running technique exercises } \\
\text { to } 20 \text { m and walking back } \\
1^{\text {st }} \text { tiptoe walking } \\
2^{\text {nd }} \text { heels walking } \\
3^{\text {rd }} \text { heel strike, put down the } \\
\text { whole foot and finish with } \\
\text { anterior foot load } \\
4^{\text {th }} \text { backward running } \\
5^{\text {th }} \text { low impact skipping } \\
6^{\text {th }} \text { backward skipping on } \\
\text { right leg } \\
7^{\text {th }} \text { backward skipping on } \\
\text { left leg } \\
8^{\text {th }} \text { wide strides with jump } \\
\text { self-righting each touchdown } \\
9^{\text {th }} \text { progressive with wide } \\
\text { strides and low frequency } \\
10^{\text {th }} \text { progressive with high } \\
\text { frequency strides and short } \\
\text { strides } \\
\text { Continuous running R1 until } \\
\text { starting to stretch. } 10 \text { ' } \\
\text { Back to calm: } \\
\text { Stretching }\end{array}$ \\
\hline
\end{tabular}




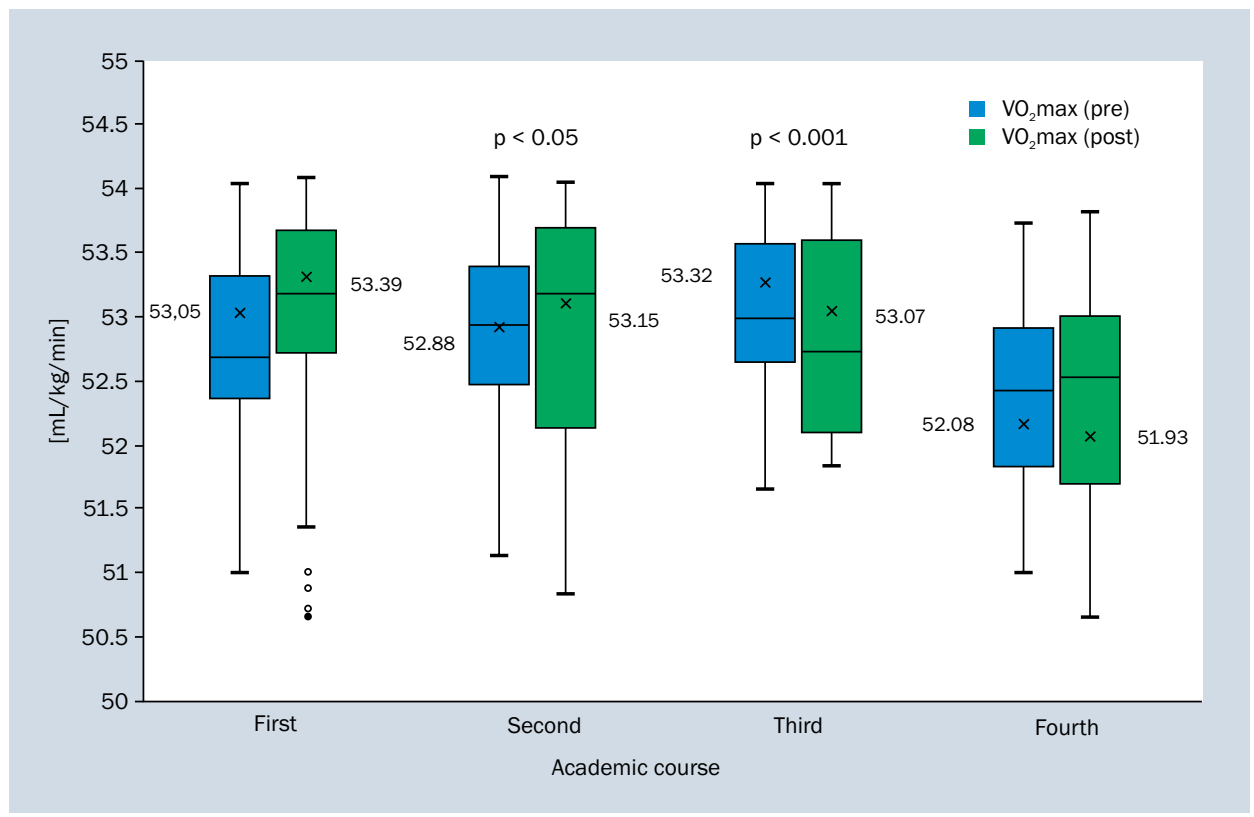

Figure 1. $\mathrm{VO}_{2}$ max level of Spanish naval cadets according to academic year (pre-post)

difficult to pass than the calisthenics portion, candidates should look to achieve 30 min per day of vigorous activity if they wish to increase their chance of passing the physical fitness test. This follows the exercise recommendations issued by the American College of Sports Medicine on improving health [4].

In the case of the physical training programme carried out at the MNAM and in response to the main objective of maintaining and training the physical and cardiovascular fitness of cadets, and preparing them for the biannual physical fitness tests, this programme is organised by giving relevance to different aspects of physical conditioning. The content of the programme focuses, in the first semester, on running training ( 3 out of 5 days), strength power circuit ( 1 day) and swimming ( 1 day). In the second semester, the programme focuses on running training ( 3 out of 5 days) and strength power circuit (2 days), without including the previous semester's specific swimming training. There does indeed seem, therefore, to be coherence between the tests used to assess physical fitness and the training programme carried out.

Emphasis in the military has traditionally been placed on the long-distance run, which is one of the simplest and cheapest forms of training that large groups can do in almost any environmental conditions, with little or no formal knowledge or understanding of the principles of physical training [7]. However, the test with the longest duration used in the current battery of tests is the $1000 \mathrm{~m}$ race, which is supposed to assess cadets' stamina, but this test is at the lower limit of assessing this data field. In other studies, the
$\mathrm{VO}_{2}$ max is assessed based on tests that include a minimum of $1600 \mathrm{~m}$ [17], in some cases up to $3000 \mathrm{~m}$ in specific physical fitness protocols [18], and in a few cases nearly $4800 \mathrm{~m}$ [10], with the most commonly adopted distance being the $2400 \mathrm{~m}$ used by the American Navy and the British Royal Navy [3, 17]. Wilkinson et al. [19] in their study observed a direct correlation between the $2400 \mathrm{~m}$ race and the multistage fitness test, these representing a practical means of assessing aerobic fitness when space is limited. But in the case of the tests carried out in the MNAM. the longest distance test used is $1000 \mathrm{~m}$ and no multistage fitness test is included. In Dada et al. [1], as the APFT was used to assess the physical fitness of the sample, a 2 min sit ups and a 2-mile (3218.69 m) run were included in the measurements. Neither the 2 min sit ups nor the 2-mile run are included in the MNAM fitness tests; however, because the longest run included in the MNAM tests is a $1000 \mathrm{~m}$ race, and performance over the two different running lengths would probably be remarkably different because they involve different patterns of energy production.

In other military forces, such as the Norwegian Navy Special Operation Forces, for example, physical training is focused on aerobic endurance, through running and local muscular endurance training (bodyweight circuit training) [7, 18], a situation similar to that carried out in the MNAM. This type of training, as is more generally reflected in different public health guidelines, is characterised by prolonged periods of continuous exercise at a moderate-intensity pace [7]. Currently, however, the Norwegian Navy Special Operation Forces are taking a new approach to conform with new 
demands and organizational modifications because it has been observed that an exclusive combination of mixed endurance-based training, without any individualization, does probably not provide the optimal stimulus to improve other capacities such as strength and power $[18,20]$. The reason for developing a new anaerobic work capacity test for the Norwegian Navy Special Operation Forces was due to the lack of a valid, specific, and easily implemented anaerobic work capacity test for its force operators in the literature. The test simulates a scenario in which an operator must run a set distance and evacuate a person by dragging them out of the "danger zone." This scenario was chosen because it could be easily replicated, and the movement-pattern, external load, and muscles recruited are similar to those necessary in many critical "anaerobic" combat situations [20].

Interval training could be adapted to conform to the reality in which the cadets present different levels of physical fitness and thus ensure that all members of a given unit benefit equally from the quality training time that is designed to prepare them for the demands of their missions [7]. In the case of the MNAM physical training programme, both the first and second semester include 1 day of interval training, which involves a reduced training volume and time when compared with traditional endurance training, as mentioned by Gibala et al. [7].

Considering upper train force, the Army Soldiers in Basic Combat Training and operational units of the USA Army participated in the study by Dada et al. [1] and the authors observed an average measurement for 2 min push ups of $34.9 \pm 14.8$ repetitions for the men of age group 17-21, and $36.3 \pm 14.8$ for the men of age group 22-26. In our study, the age groups analysed were different from those in the study by Dada et al. [1], but independently, higher values were observed, with average values of $42.8 \pm 10.8$ and $42.5 \pm 11.9$ recorded for age groups $18-19$ and $20-21$, respectively, and $42.6 \pm 11.1$ and $42.7 \pm 10.7$ for age groups 22-23 and > 24, respectively, though in our case, in the analysis by age group both men and women were included.

In the study by Groeller et al. [8] carried out on a sample of 51 Australian soldiers from 5 different specialities (infantry, armoured, combat engineers, artillery and transport trades) during their basic military training and initial employment training, different physical aspects were assessed, including 2 min push ups and vertical jump. In the case of the vertical jump measurement, data was taken using a portable force platform and the force applied was measured in $\mathrm{N}$, this being different from the methodology employed in the current work and making data comparison impossible. However, measurement on 2 min push ups repetitions reached similar values to ours in the first assessment: $41.1 \mathrm{~cm}(37.5-44.7)$, but values observed after the basic military training: $49.7 \mathrm{~cm}(47.0-52.4)$ and after initial employment training: $57.5 \mathrm{~cm}$ (54.4-60.6), were superior than those observed for our sample: $(42.7 \pm 11.8)$, a fact which could be explained by more focussed training on this specific ability.

Even when we considered other military naval forces, we failed to find any bibliographical evidence of other physical fitness protocols which include swimming in their tests. It is important to stress that we did not consider this factor because, in the case of the MNAM, they do not take physical performance in swimming to be critical: their physical training protocol only includes swimming once a week in the first semester; the $50 \mathrm{~m}$ swimming test has a cut off time to pass of over $50 \mathrm{~s}$, and the average times achieved by the cadets is around $35 \mathrm{~s}$.

Finally, a limitation to the internal validity of this study lies in the fact that some of the cadets do not perform in the tests at their maximum capacity because those who reach the minimum marks required for each academic year do not necessarily seek the best performance in the different tests. In addition, another limiting aspect when it comes to being able to trace and relate the physical condition of MNAM cadets is that their $1000 \mathrm{~m}$ test differs from other armies that focus their endurance tests on distances close

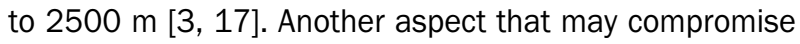
the external validity of our study is the fact that the protocol described for the evaluation of vertical jumping, for example, and that's still used in the Spanish Army are not currently those used in the sports and health field and it would be necessary to update the protocol and tool to be used to enable comparison with samples of similar characteristics (other military personnel, local police, firefighters, ...); however, not committing the internal validity of the same. However, it should be noted that access to the important sample of MNAM and the knowledge of its training protocol enables the initial recognition of possible adaptations necessary in both the physical condition evaluation protocol and the training protocol itself in the near future.

\section{CONCLUSIONS}

The level of physical fitness of cadets at the MNAM appears to be at an acceptable level when compared to that of other countries' military naval corps. However, it is of importance to consider adjusting the physical training protocol so it is more in line with the fitness tests employed and the subsequent demands that such military personnel will face in garrison and deployed environments.

The authors' point is that not only is it important to assess the physical fitness of military personnel on the basis of the tests included in the Physical Fitness Testing Protocol, but also to adapt to new situations as they arise and to stop and reflect on whether the tests currently being used assess the aspects of physical fitness that they should. 


\section{ACKNOWLEDGEMENTS}

The authors declare that they have no competing interests and that the research has not received any funding. The authors thank all the cadets who have voluntarily participated in the study, the leadership of the MNAM and the officer responsible for the "Admiral Álvarez-Ossorio" endowed chair.

\section{AVAILABILITY OF DATA AND MATERIALS}

The datasets generated and analysed during the current study are not publicly available as they considered to be national documents.

\section{CONFLICT OF INTEREST}

The authors declare that they have no competing interests.

\section{REFERENCES}

1. Dada EO, Anderson MK, Grier T, et al. Sex and age differences in physical performance: A comparison of Army basic training and operational populations. J Sci Med Sport. 2017; 20 Suppl 4: S68-S73, doi: 10.1016/j.jsams.2017.10.002, indexed in Pubmed: 29100826.

2. Anderson MK, Grier T, Canham-Chervak M, et al. Effect of mandatory unit and individual physical training on fitness in military men and women. Am J Health Promot. 2017; 31(5): 378-387, doi: 10.1177/0890117116666977, indexed in Pubmed: 27630111.

3. Sargent C, Lacey S, Gebruers C, et al. The development and optimisation of a quantitative physical fitness scoring system for use amongst Naval Service personnel. Int Marit Health. 2016; 67(3): 171-178, doi: 10.5603/IMH.2016.0032, indexed in Pubmed: 27681218.

4. Cuddy JS, Slivka DR, Hailes WS, et al. Factors of trainability and predictability associated with military physical fitness test success. J Strength Cond Res. 2011; 25(12): 3486-3494, doi: 10.1519/ JSC.0b013e318217675f, indexed in Pubmed: 22080323.

5. Warr BJ, Scofield DE, Spiering BA, et al. Influence of training frequency on fitness levels and perceived health status in deployed National Guard soldiers. J Strength Cond Res. 2013; 27(2): 315-322, doi: 10.1519/JSC.0b013e31827e1347, indexed in Pubmed: 23222077.

6. Tingelstad HC, Theoret D, Spicovck M, et al. Explaining performance on military tasks in the Canadian armed forces: the importance of morphological and physical fitness characteristics. Mil Med. 2016; 181(11): e1623-e1629, doi: 10.7205/MILMED-D-15-00458, indexed in Pubmed: 27849498.

7. Gibala MJ, Gagnon PJ, Nindl BC. Military applicability of interval training for health and performance. J Strength Cond Res. 2015; 29 Suppl 11: S40-S45, doi: 10.1519/JSC.0000000000001119, indexed in Pubmed: 26506197.

8. Groeller H, Burley S, Orchard P, et al. How effective is initial military-specific training in the development of physical performance of soldiers? J Strength Cond Res. 2015; 29 Suppl 11: S158-S162, doi: 10.1519/JSC.0000000000001066, indexed in Pubmed: 26506181.

9. Bilzon Jப, Scarpello EG, Bilzon E, et al. Generic task-related occupational requirements for Royal Naval personnel. Occup Med (Lond). 2002; 52(8): 503-510, doi: 10.1093/occmed/52.8.503, indexed in Pubmed: 12488523.

10. Bartlett JL, Phillips J, Galarneau MR. A descriptive study of the U.S. Marine Corps fitness tests (2000-2012). Mil Med. 2015; 180(5): 513-517, doi: 10.7205/MILMED-D-14-00490, indexed in Pubmed: 25939104.

11. Mackey CS, DeFreitas JM. A longitudinal analysis of the U.S. Air Force reserve officers' training corps physical fitness assessment. Mil Med Res. 2019; 6(1): 30, doi: 10.1186/s40779-019-0219-4, indexed in Pubmed: 31543076.

12. Wyss T, Von Vigier RO, Frey F, et al. The Swiss Army physical fitness test battery predicts risk of overuse injuries among recruits. J Sports Med Phys Fitness. 2012; 52(5): 513-521, indexed in Pubmed: 22976738.

13. Williamson DA, Bathalon GP, Sigrist LD, et al. Military services fitness database: development of a computerized physical fitness and weight management database for the U.S. Army. Mil Med. 2009; 174(1): 1-8, doi: 10.7205/milmed-d-03-7807, indexed in Pubmed: 19216292.

14. Ross WD, Marfell-Jones MJ. Cineantropometría. In: Macdougall DJ, Wenger HA, editors. Evaluación fisiológica del deportista. Paidotripo, Barcelona 2013.

15. MADOC Espa a. MV3-101. Manual del Sistema de Evaluación Física Individual del Ejército de Tierra. 2010.

16. Grier TL, Canham-Chervak M, Bushman TT, et al. Evaluating injury risk and gender performance on health- and skill-related fitness assessments. J Strength Cond Res. 2017; 31(4): 971-980, doi: 10.1519/JSC.0000000000001805, indexed in Pubmed: 28328715.

17. Lunt H, Roiz De Sa D, Roiz De Sa J, et al. Validation of one-mile walk equations for the estimation of aerobic fitness in British military personnel under the age of 40 years. Mil Med. 2013; 178(7): 753-759, doi: 10.7205/MILMED-D-12-00369, indexed in Pubmed: 23820349.

18. Solberg PA, Paulsen G, Slaathaug OG, et al. Development and implementation of a new physical training concept in the Norwegian navy special operations command. J Strength Cond Res. 2015; 29 Suppl 11: S204-S210, doi: 10.1519/JSC.0000000000001085, indexed in Pubmed: 26506189.

19. Wilkinson DM, Blacker SD, Richmond VL, et al. Relationship between the 2.4-km run and multistage shuttle run test performance in military personnel. Mil Med. 2014; 179(2): 203-207, doi: 10.7205/ MILMED-D-13-00291, indexed in Pubmed: 24491618.

20. Angeltveit A, Paulsen G, Solberg PA, et al. Validity, reliability, and performance determinants of a new job-specific anaerobic work capacity test for the Norwegian navy special operations command. J Strength Cond Res. 2016; 30(2): 487-496, doi: 10.1519/ JSC.0000000000001041, indexed in Pubmed: 26815177. 